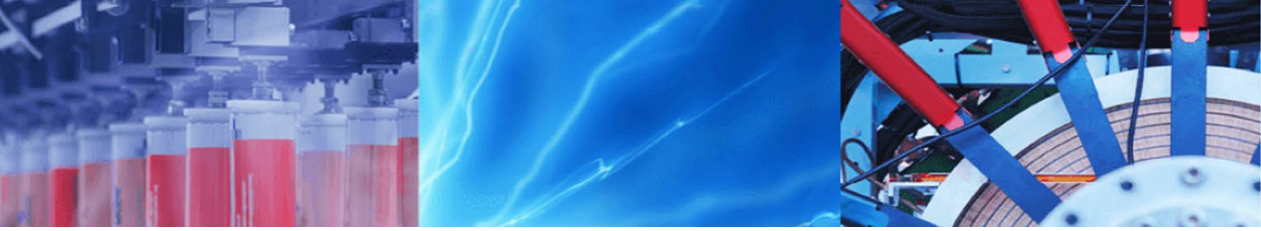

Research Article

\title{
Effective enhancement of dielectric properties in cold-pressed polyvinyledene fluoride/barium titanate nanocomposites
}

\author{
Maheswar Panda ${ }^{1}$ (D) A Avaneesh Mishra ${ }^{1} \cdot$ Prashant Shukla $^{1}$
}

(c) Springer Nature Switzerland AG 2019

\begin{abstract}
The dielectric behaviour of a polymer/ceramic composite (PCC) based on ferroelectric polymer [polyvinylidene fluoride (PVDF)] and nano-barium titanate $\left(n-\mathrm{BaTiO}_{3}\right)$, prepared under the novel cold pressing method, was investigated. Large enhancement of effective dielectric constant $\left(\varepsilon_{\text {eff }} \sim 450\right)$ with lower loss tangent ( $\left.\tan \delta \sim 0.9\right)$ at $50 \mathrm{~Hz}$ was observed for the PCC with 0.60 as the volume fraction of $n-\mathrm{BaTiO}_{3}$ into the PVDF matrix. The enhancement of $\varepsilon_{\text {eff }}$ is attributed to the large interfacial polarisation arising due to the charge storage at the interfaces of the spherulites of PVDF and at the polymer/filler interfaces of PCC. The dielectric results have been explained on the basis of sum effect with the help of the standard Yamada model at $1 \mathrm{kHz}$, while the low-frequency dielectric behaviour has been explained qualitatively as a combination of both interfacial polarisation at the interfaces of the spherulites and the sum effect of the Yamada model. The achieved lower $\tan \delta$ for the PCC as compared to the polymer/metal composites (PMC) is attributed to the highly insulating nature of PVDF and semiconducting $n-\mathrm{BaTiO}_{3}$. The thermal stability of the composites is also maintained due to the higher melting temperature $\left(170^{\circ} \mathrm{C}\right)$ of PVDF. The cold-pressed PCC based on PVDF will act as better polymer dielectric for electrical energy storage applications as compared to the reported cold-pressed PMC and the cold pressing may be an effective method of preparation (since the unnecessary introduction of thermal disorder is avoided in this method) in developing polymer dielectrics.
\end{abstract}

Keywords Polymer dielectrics · Spherulites · Ferroelectric polymers · Barium titanate · Dielectric constant · Loss tangent $\cdot$ Polymer nanocomposites

\section{Introduction}

Polymer dielectrics (PD) with higher energy density and lower loss tangent $(\tan \delta$ ) with higher breakdown field strength are the emerging materials of future for electrostatic energy storage applications due to their flexibility, non-toxicity, bio-compatibility, low cost, higher visco-elastic properties, etc. [1-12]. For this application, the maximum stored energy density is an important parameter, and for a linear dielectric, the maximum stored energy per unit volume is $U=\frac{1}{2} K \varepsilon_{0} E_{\text {max }}^{2}$, where $K$ is the relative dielectric constant and $E_{\max }$ is the maximum electric field, which can be applied to the material (proportional to the breakdown field of the material) [4-12]. In development of these PD, over the last two decades of research, the ferroelectric polymers, e.g. polyvinyledene fluoride (PVDF) matrix, have been preferred due to its high static dielectric constant $(\sim 15)$ and higher visco-elastic properties and higher insulating nature as compared to other non-polar polymers [1-27]. The varieties of fillers, such as micro- and nanoparticles of metal, ceramic, carbon nanotube, carbon black, graphite, graphene, conducting, and polymer, are under continuous use in the development of these PD [1-31]. However, the development of the polymer/ ceramic composites (PCC) has been dormant over the

Maheswar Panda, panda.maheswar@gmail.com | 'Department of Physics, Dr. Harisingh Gour Vishwavidyalaya (A Central University), Sagar, MP, India.

SN Applied Sciences (2019) 1:230 | https://doi.org/10.1007/s42452-019-0234-9

Received: 1 October 2018 / Accepted: 6 February 2019 / Published online: 13 February 2019 
last decade of research, as the effective dielectric constant $\left(\varepsilon_{\text {eff }}\right)$ for these composites was found to be very low, i.e. $\varepsilon_{\text {eff }} \sim 100$, at low frequencies due to the low dielectric constant of the polymers, as well as due to the conventional hot moulding process conditions [27-31]. In the preparation of these PCC, a very high-dielectricconstant ferroelectric ceramic, such as PMN-PT, $\mathrm{BaTiO}_{3}$, and $\mathrm{PbTiO}_{3}$, with particles of size of micron order is used in order to have improvement in the value of $\varepsilon_{\text {eff }}$ with lower $\tan \delta$. However, due to the low dielectric constant of the polymers, even with PVDF matrix (whose static dielectric constant $\sim 17$ ), much higher value of $\varepsilon_{\text {eff }}$ with lower $\tan \delta$ could not be achieved. Hence, in order to have large interfacial polarisation, the nanoparticles of the same ferroelectric ceramics also have been used into the PVDF matrix through hot moulding, and partially the approach becomes effective in order to get better PD [27-31].

In the development of these PCC based on PVDF, the traditional mixed technique (solution casting followed by hot moulding) is used, during which the spherulites of PVDF get lost [32], which results in getting the lower value of $\varepsilon_{\text {eff }}$. Recently, our group has developed the cold pressing technique in preparing the PD based on PVDF, due to which the spherulites [32] of PVDF are retained, and they help in the additional storage of electrical charge due to the created additional interfaces, which results in higher interfacial polarisation, and hence, the higher value of $\varepsilon_{\text {eff }}$ is achieved [5, 13-17].

In developing these PD, among the variety of fillers, except the insulating ceramic fillers, mostly the polymer/ metal or conductor composites (PMC) have undergone research, in the last two decades by various research groups [7-26]. The origin of interest in these PMC is that, in these composites a very high value of $\varepsilon_{\text {eff, }}$ is achieved due to the percolative second-order insulator to metal transition (IMT) at the percolation threshold $\left(f_{\mathrm{c}}\right)$. At $f_{\mathrm{c}}$, the $\varepsilon_{\text {eff }}, \tan \delta$, and effective ac conductivity $\left(\sigma_{\text {eff }}\right)$ undergo sharp increment due to the IMT and $f_{\mathrm{c}}$ becomes the point of discontinuity for $\varepsilon_{\text {eff }}$ in the case of PMC [33]. However, the main drawback of these PMC, which inhibits them from their technological applications, is the unwanted higher value of $\tan \delta$ and $\sigma_{\text {eff }}$.

Hence, in order to overcome the major difficulty of these PD (higher value of $\tan \delta$ ) based on PMC, the interest on the PCC-based PD should be focused with novel method of preparation in order to have lower value of $\tan \delta$ along with the higher value of $\varepsilon_{\text {eff }}$ One of the very simple methods in finding the true percolation behaviour of PMC is the preparation of cold-pressed composites by Panda et al. [5, 13-17], in which the spherulites of PVDF are retained and higher $\varepsilon_{\text {eff }}$ is observed due to the additional storage of electrical charge at the interfaces of the spherulites of the composites.

Hence, we do believe that by changing the traditional process condition (hot moulding of the thick films prepared from solution casting) to the cold pressing method, developed by our group, in which the spherulites of PVDF will be retained for the case of PCC (like that of the PMC), definitely higher value of $\varepsilon_{\text {eff }}$ will be achieved [5, 13-17], with lower $\tan \delta$ due to the high-dielectric-constant insulating ferroelectric ceramics. Hence, in order to develop a PCC with high $\varepsilon_{\text {eff }}$ and lower $\tan \delta$, ferroelectric polymer (PVDF) and high-dielectric-constant nano-ceramic $\left(n-\mathrm{BaTiO}_{3}\right.$ ) with particle size $\sim 100 \mathrm{~nm}$ (having large surface area) were selected, and the prepared composites have undergone the microstructural, dielectric, and electrical conductivity measurement for finding their suitability of applications.

\section{Experimental details}

A series of PCC based on PVDF with varying volume fraction of $n-\mathrm{BaTiO}_{3}\left(f_{\mathrm{BaTiO} 3}\right)$ from 0.0 to 0.60 were prepared. The composite powders of PVDF (Alfa Aesar) and the $n-\mathrm{BaTiO}_{3}$ (Aldrich) with particle size $<100 \mathrm{~nm}$ were prepared with different $f_{\mathrm{BaTiO} 3}$ by mixing with agate mortar/pestle for $2 \mathrm{~h}$, and the final samples were taken in the form of pellets under room temperature consolidation at a pressure of $30 \mathrm{MPa}$ with the help of a hydraulic press. The microstructure investigation on the samples was carried out with the help of FESEM from FEI Company of USA (SE Asia) Pte, Ltd. (Model No. SEM 450). The electrical measurements were made on all the PCC in the frequency range of $50 \mathrm{~Hz}$ to $5 \mathrm{MHz}$ and in the temperature range of room temperature to $100^{\circ} \mathrm{C}$ with the help of the LCR HiTESTER (Model No. Hioki 3520). The dielectric results of the PCC have been understood with the help of Yamada model fitted with the help of the software Mathematica.

\section{Results and discussion}

\subsection{Microstructure}

The FESEM micrographs of the cold-pressed pure PVDF (Fig. 1a, b) and PVDF/n-BaTiO ${ }_{3}$ composites with different $f_{\mathrm{BaTiO} 3}$ up to 0.60 are shown in Fig. $1 \mathrm{c}$-f. Figure $1 \mathrm{a}$, $b$ shows the presence of spherulites (the spherical semi-crystalline regions of the polymer) in the coldpressed PVDF, while both the spherulites and large created unfilled like structures (due to the cold pressing) are observable from the micrographs (Fig. 1c-f). The 

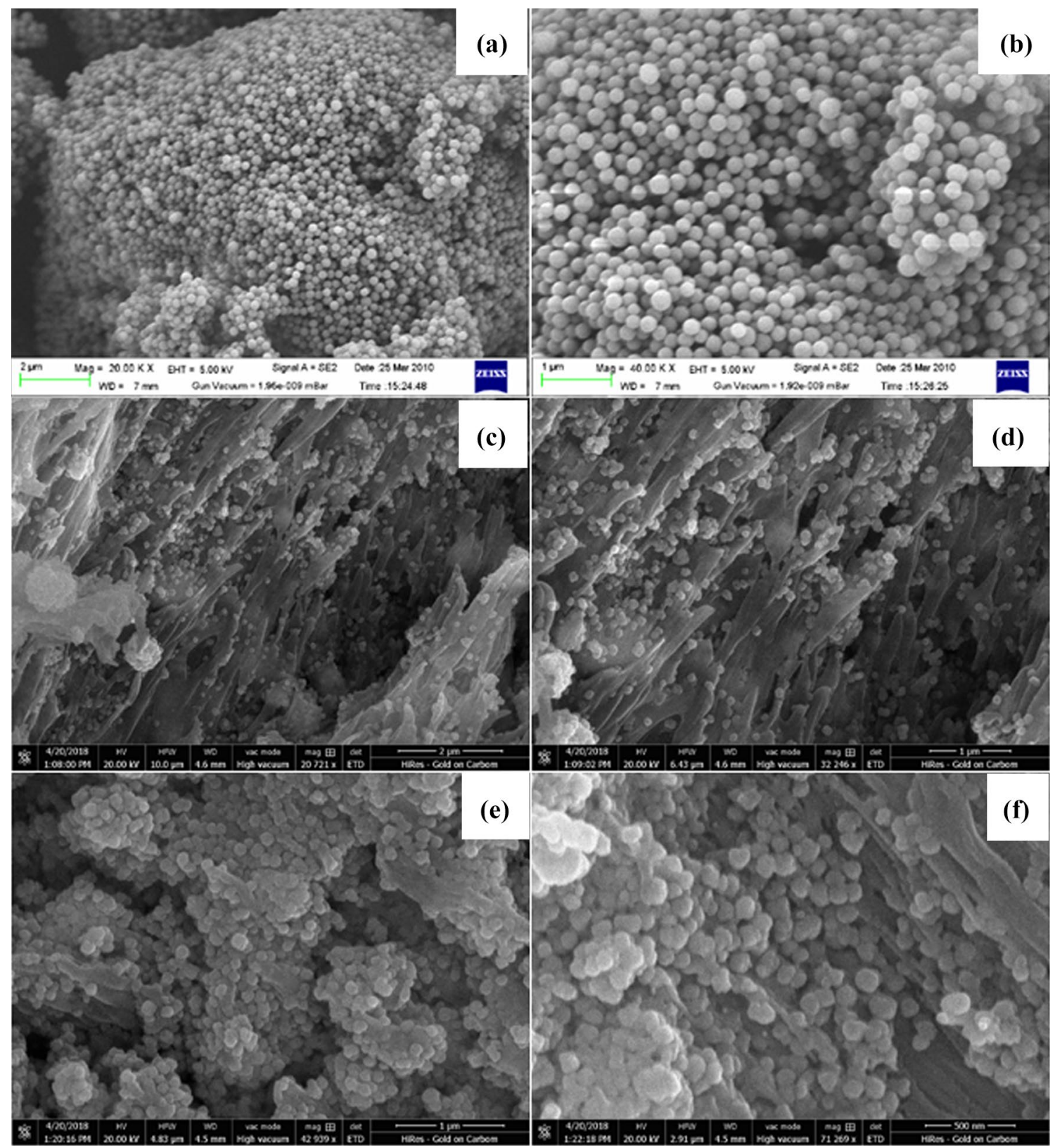

Fig. 1 FESEM micrographs of cold-pressed PCC. a Pure PVDF (lower resolution), b pure PVDF (higher resolution), $\mathbf{c} f_{\mathrm{BaT} \text { iO3 }}=0.2$ (lower resolution), $\mathbf{d} f_{\mathrm{BaTiO} 3}=0.2$ (higher resolution), e $f_{\mathrm{BaTiO} 3}=0.6$ (lower resolution), and $\mathbf{f} f_{\mathrm{BaTiO} 3}=0.6$ (higher resolution)

diameter of the spherulites present in the polymer is of the order of $\sim 0.1 \mu \mathrm{m}$ (Fig. 1a, b). Interestingly, the $n$ - $\mathrm{BaTiO}_{3}$ taken is of size $100 \mathrm{~nm}$, i.e. $0.1 \mu \mathrm{m}$, of the order of diameter of the spherulites. During cold pressing, the $n$ - $\mathrm{BaTiO}_{3}$ clusters may have taken the shape (Fig. 1c-f) inside the polymer matrix, which is of large benefit in the storage of electrical charge. From all the micrographs, it is clear that a various types of interfaces have appeared in the composites and are useful in the storage of electrical charge at the interfaces.

\subsection{Dielectric properties}

The variation of $\varepsilon_{\text {eff }}$ and $\tan \delta$ of the prepared series of PCC as a function of frequency at $300 \mathrm{~K}$ is shown in Fig. 2a, b, respectively. The value of $\varepsilon_{\text {eff }}$ at $50 \mathrm{~Hz}$ for the 0.0 sample is 16 , while this value increases up to 120 linearly up to the PCC with $f_{\mathrm{BaTiO} 3}=0.5$ and after that it increases up to the value of 330 and 420 for the samples with $f_{\mathrm{BaTiO} 3}=0.55$ and $f_{\mathrm{BaTiO} 3}=0.60$, respectively. The higher value of $\varepsilon_{\text {eff }}$ for the $f_{\mathrm{BaTiO} 3}=0.55$ and $f_{\mathrm{BaTiO} 3}=0.60$ is due to the large interfacial polarisation arising both due to the presence 


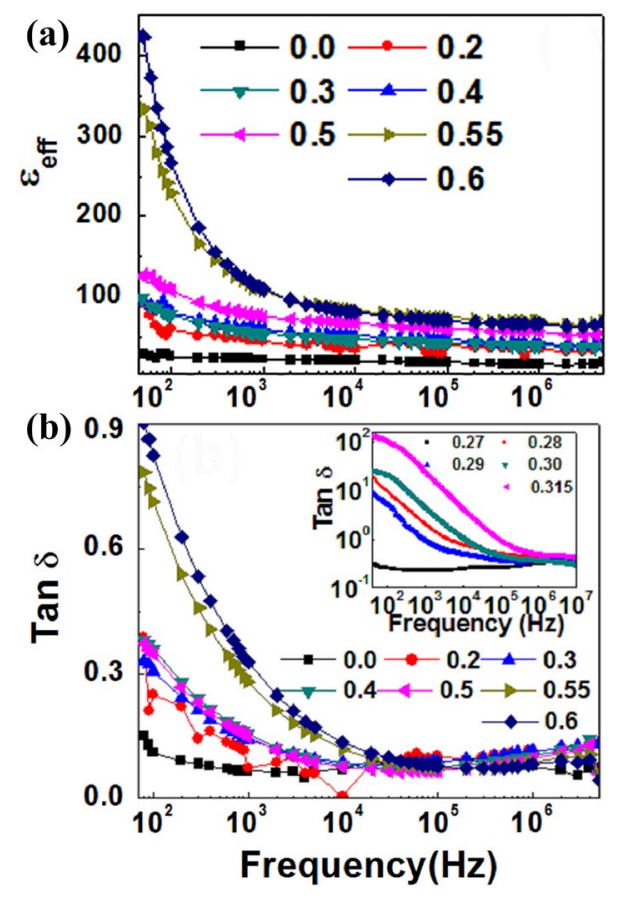

Fig. 2 Variation of $\mathbf{a} \varepsilon_{\text {eff }}$ and $\mathbf{b} \tan \delta$ as a function of frequency at $300 \mathrm{~K}$ for the PD, Inset: $\tan \delta \sim$ frequency for some typical percolative PMC samples showing higher $\tan \delta[13]$

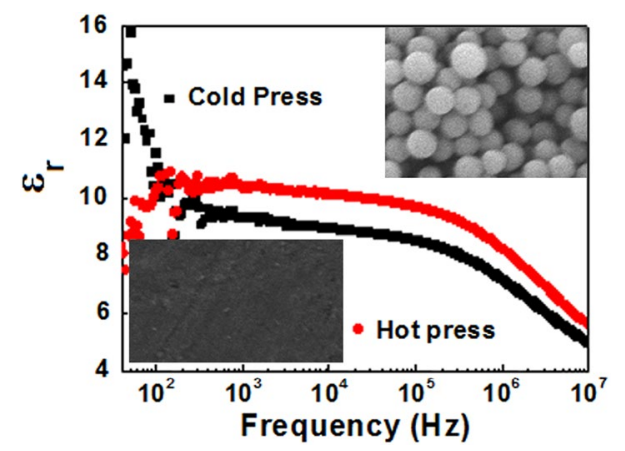

Fig. 3 Variation of dielectric constant $\left(\varepsilon_{\mathrm{r}}\right)$ with frequency for both cold and hot press PVDF, Inset: the FESEM micrograph of the cold/ hot-moulded PVDF showing the presence/loss of spherulites at temperature higher than the room temperature

of spherulites and created large interface like structures during cold pressing, while the spherulites are always lost for the hot-moulded samples (Fig. 3).

Figure 3 shows the static dielectric constant $\left(\varepsilon_{\mathrm{r}}\right)$ of the cold-pressed pure PVDF is $\sim 16$, i.e. higher than the $\varepsilon_{\mathrm{r}}$ of hot-moulded pure PVDF $(\sim 10)$ due to the loss of spherulites (Inset, Fig. 3) of the polymer. The value of $\varepsilon_{\text {eff }}$ decreases with increase in frequency for all the PCC due to the absence of contribution from interfacial polarisation at higher frequencies where only the contribution from dipolar and atomic polarisation exists. A very low $\tan \delta$ for the
PCC with $f_{\mathrm{BaTiO} 3}=0.6$ (having highest $\varepsilon_{\text {eff }}=420$ observed at $50 \mathrm{~Hz}$ ) was found to be only 0.9 at $50 \mathrm{~Hz}$ and that value also decreases with increase in frequency, and the trend of decrement is also observed for all the PCC. However, in the recently reported cold-pressed PMC [13], the $\tan \delta$ value was reported to be 10 at $50 \mathrm{~Hz}$ (Inset, Fig. 2b) for the percolative sample, with $\varepsilon_{\text {eff }} \sim 2000$. Hence, the PMC at $f_{c}$ shows ten times higher value of $\tan \delta$ in comparison with the result of PCC, although both types of polymer composites are prepared by the same cold pressing procedure.

The $\varepsilon_{\text {eff }} \sigma_{\text {eff }}$ and $\tan \delta$ of the composites as a function of $f_{\mathrm{BaTiO}}$ at different frequencies are shown in Fig. 4. The $\varepsilon_{\text {eff }}$ rises linearly from 16 to 120 when $f_{\mathrm{BaT} \text { iO3 }}$ increases from 0.0 to 0.50 at $100 \mathrm{~Hz}$, while due to the large interfacial polarisation occurring due to the presence of spherulites and interfaces at the PCC, the $\varepsilon_{\text {eff }}$ increases largely from 120 to 350 and 420 for $f_{\mathrm{BaTiO} 3}=0.55$ and 0.60 , respectively. It was found that the expression developed by Yamada et al. (which is a model for explaining the sum properties of the composite) can fit the dielectric data very well (Fig. 4b) at frequency $1 \mathrm{kHz}$ or above. The model is given by

$\varepsilon_{\text {eff }}=K_{\mathrm{PVDF}}\left[1+\frac{n f_{\mathrm{BaTiO}_{3}}\left(K_{\mathrm{BaTiO}_{3}}-K_{\mathrm{PVDF}}\right)}{n K_{\mathrm{PVDF}}+\left(K_{\mathrm{BaTiO}_{3}}-K_{\mathrm{PVDF}}\right)\left(1+f_{\mathrm{BaTi}_{3}}\right)}\right]$,

where $\varepsilon_{\text {eff }}$ is the effective dielectric constant of the composite, $K_{\mathrm{PVDF}}$ and $\mathrm{K}_{\mathrm{BaTiO}_{3}}$ are the dielectric constants of the polymer matrix and the ceramic, respectively, $\mathrm{BaTiO}_{3}$ is the volume fraction of the ceramic and ' $n$ ' is a parameter related to the geometry of ceramic particles [23]. The values of $K_{\mathrm{PVDF}}, K_{\mathrm{BaTiO}_{3}}$ and $n$ obtained from the fitting of Eq. (1) to the experimentally measured dielectric data of the composites are 17,1600, and 10, which are in good agreement with the earlier literature $[n=10.6$ for PVDF/PZT composites, that found in Ref. 22]. The important point we would like to communicate here is that the Yamada model could not be fitted with the experimental dielectric data of $100 \mathrm{~Hz}$ or low frequencies, as the additional contributions of electrical charge (in addition to the presence of sum properties of both the components) at the interface of the spherulites (due to cold pressing) give rise to rapid enhancement of $\varepsilon_{\text {eff }}$. The analysis suggests that a modified Yamada model may be developed at low frequencies, to account for the contribution of both sum properties and spherulites, which remained as a challenge to take care of the spherulites in this type of cold-pressed PCC. It is important to point out here that, it should be a challenge for theoretical work not only for this investigated case of PCC but also for any other PCC containing spherulites [i.e. containing some additional interfacial polarisation over the conventional sum effect] to be established as a general work. 
Fig. 4 Variation of a $\varepsilon_{\text {eff }}$ experimentally, $\mathbf{b}$ fitting of $\varepsilon_{\text {eff }}$ experimental data at $1 \mathrm{kHz}$ with Yamada model as a function of $f_{\mathrm{BaTiO}}, \mathbf{c} \sigma_{\text {eff }}$ and $\mathbf{d} \tan \delta$ as a function of $f_{\mathrm{BaTiO} 3}$ for various frequencies at $300 \mathrm{~K}$
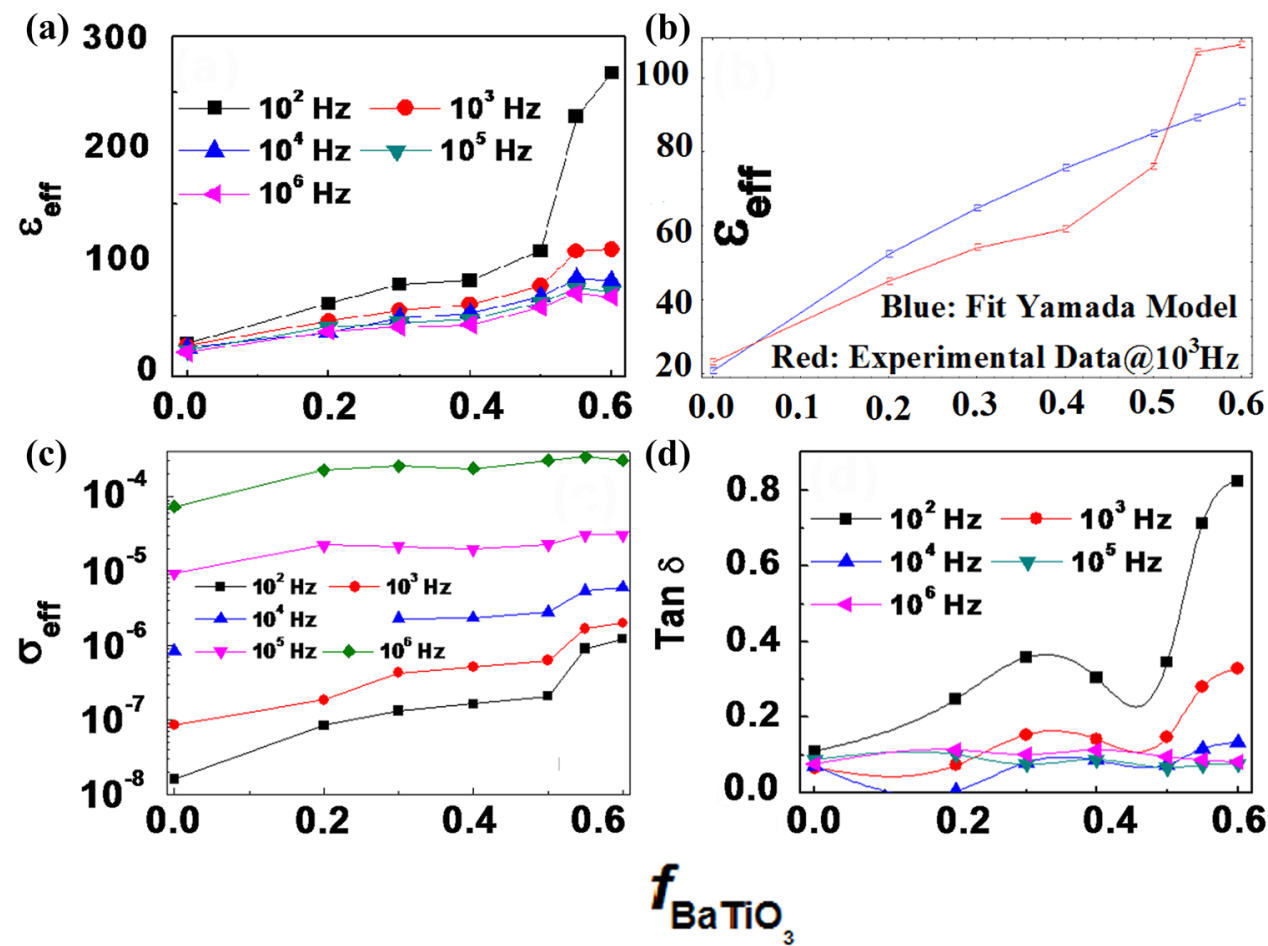

The $\sigma_{\text {eff }}$ and $\tan \delta$ for all the composites increase with the increase in $\mathrm{BaTiO}_{3}$ in the PCC slowly, suggesting the semiconducting nature of the $\mathrm{BaTiO}_{3}$ nano-ceramics, thereby becoming very helpful in the reduction in tan $\delta$ with achieved higher $\varepsilon_{\text {eff }}(\sim 400)$. With $f_{\mathrm{BaTiO}_{3}}=0.6$, the $\sigma_{\text {eff }}$ value varies within $10^{-8}-10^{-4} \Omega^{-1} \mathrm{~cm}^{-1}$ for frequency varying between $50 \mathrm{~Hz}$ and $5 \mathrm{MHz}$, while the value of $\tan$ $\delta$ is maintained in between 0.1 and 0.9. The value of $\sigma_{\text {eff }}$ and $\tan \delta$ is also found to be increasing with increase in frequency, suggesting primarily the hopping type of $A C$ conduction in the PCC. Similarly, $\sigma_{\text {eff }}$ value for all the PCC was found to be very low, i.e. less than $10^{-4} \Omega^{-1}$ for all the PCC and that value also almost remains constant over the entire frequency range. The $\tan \delta$ increases slowly as a function of $f_{\mathrm{BaTiO} 3}$ and was found to be less than 0.9 even with the PCC having $f_{\mathrm{BaTiO} 3}=0.6$.

The thermal stability of the PCC was checked by measuring the electrical parameters as a function of temperature and is given in Fig. 5. It was found that for $f_{\mathrm{BaTiO} 3}=0.4$ (Fig. 5a) and $f_{\mathrm{BaTiO} 3}=0.50$ (Fig. 5b), the low-frequency $(50 \mathrm{~Hz})$ value of $\varepsilon_{\text {eff }}$ is maintained at a thermal stabilised value of 90 and 130 (with their corresponding decrement as a function of frequency) as a function of temperature from 40 to $100{ }^{\circ} \mathrm{C}$. The stabilisation of $\varepsilon_{\text {eff }}$ is attributed to the major effective contribution coming from the sum properties of the dielectric constant of both the components. However, for the samples with $f_{\mathrm{BaTiO} 3}=0.55$ and 0.6 , the reached $\varepsilon_{\text {eff }} \sim 350$ and 420 value arises due to the sum properties of the dielectric constant of both the components and also due to the major contribution of the spherulites. Hence, with the increase in temperature, the $\varepsilon_{\text {eff }}$ decreases due to the diminishing of the spherulites of the PCC (Fig. 5c, d). Hence, the spherulites are always useful at room temperature in case of PCC for achieving high value of $\varepsilon_{\text {eff }}$ with lower $\tan \delta$.

\subsection{Electrical conductivity}

The variation of ac conductivity (which has been calculated as $\sigma_{\mathrm{ac}}=\omega \varepsilon_{0} \varepsilon \tan \delta$ ) as a function of frequency (conductivity spectrum) at different $f_{\mathrm{BaTiO} 3}$ is shown in Fig. 6 . The $\sigma_{\text {eff }}$ as a function of frequency was found to be the hopping type of conductivity satisfying the Johnscher's fractional power law of AC conduction [34-36]. It can be observed that the plot shows dispersion of ac conductivity with frequency for all the PCC corresponding to $f_{\mathrm{BaTiO} 3} \leq 0.20$, agrees very well with Eq. (2), i.e.

$\sigma_{\mathrm{ac}}(\omega)=\sigma_{\mathrm{dc}}+A \omega^{k}$,

with the $\sigma_{\mathrm{dc}}$ part becoming zero and the value of $k \sim 1$. The absence of dc conductivity for the samples with $f_{\mathrm{BaTiO}_{3}} \leq 0.20$ can be interpreted as the non-presence of percolating paths (being formed from the semiconducting $\mathrm{BaTiO}_{3}$ nano-ceramics in the PVDF matrix) due to the insufficient $f_{\mathrm{BaTiO} 3}$. It can also be observed that the longrange dc conduction starts to appear for $f_{\mathrm{BaTiO} 3}=0.3-0.5$, but a good fit of Eq. (2) could not be obtained for them as the percolating paths were not sufficient. But interestingly, 
Fig. 5 Variation of $\varepsilon_{\text {eff }}$ as a function of frequencies for the temperature varying from 40 to $100^{\circ} \mathrm{C}$ for varying $f_{\mathrm{BaTiO} 3}$. a 0.40 , b 0.50, c 0.55 , and d 0.60

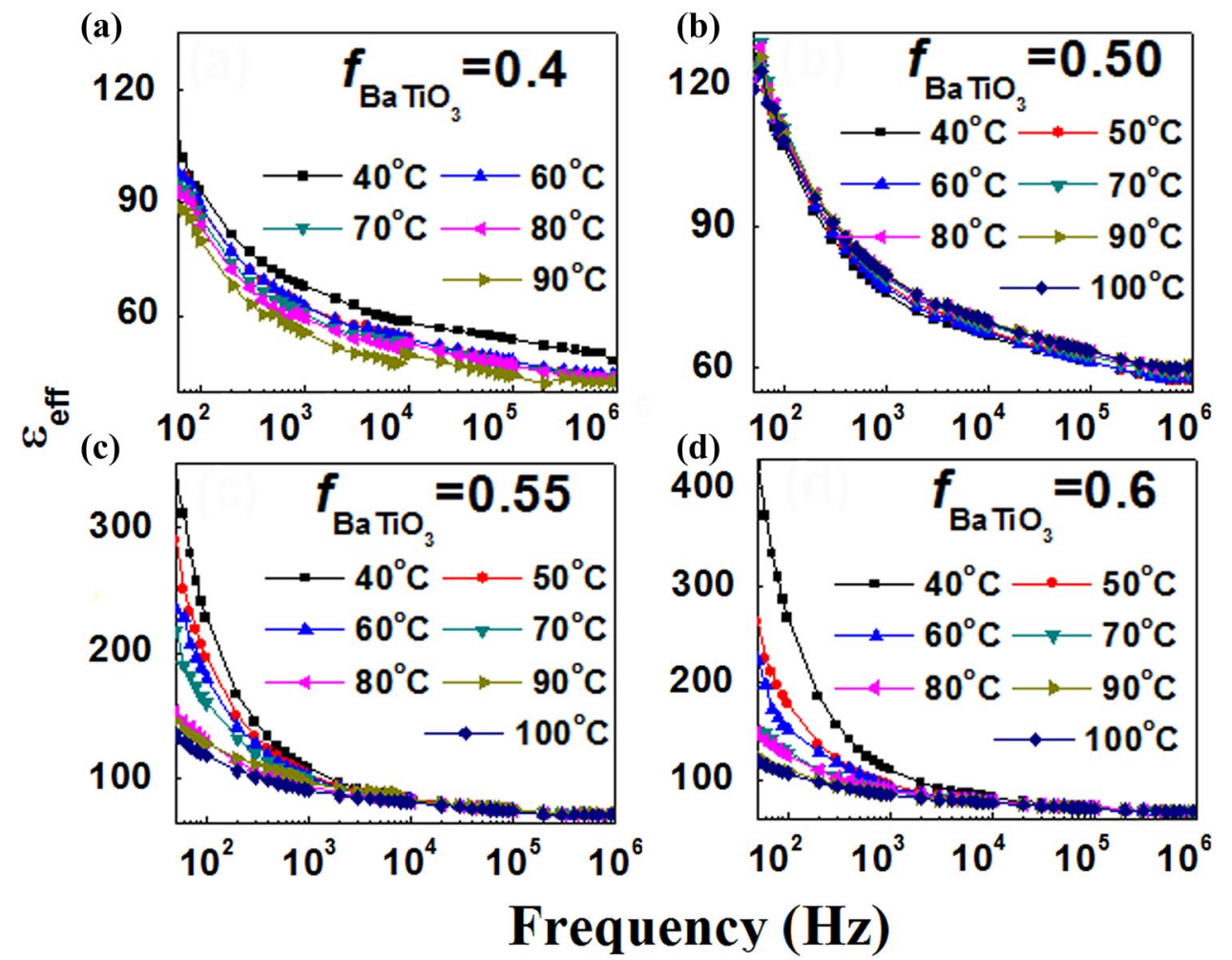

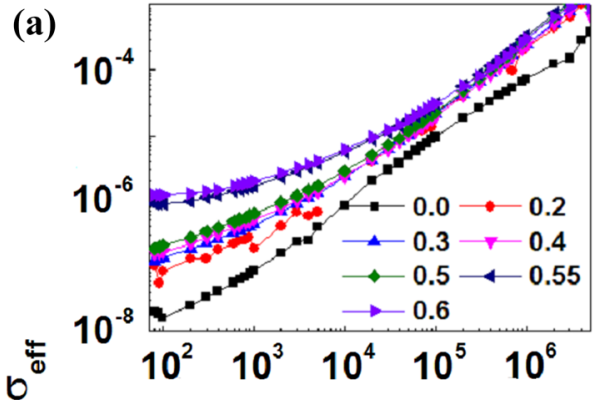

(b)

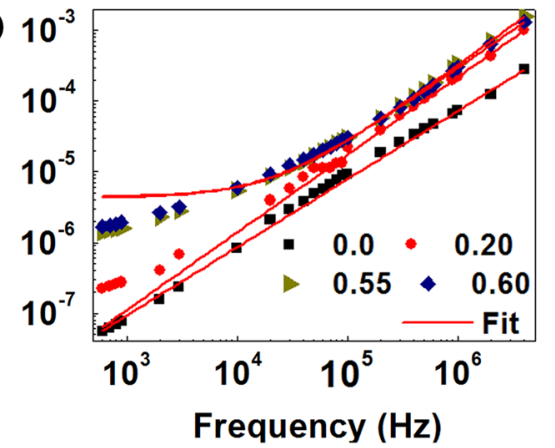

Fig. 6 Variation of $\mathbf{a} \sigma_{\text {eff }}$ experimentally and $\mathbf{b} \sigma_{\text {eff }}$ fitted with Johnscher's Power law, as a function of frequency

for the samples with $f_{\mathrm{BaTiO} 3} \geq 0.55$, a mixed conductivity is found, i.e. a dc plateau up to a certain frequency, separated by ac conductivity beyond that frequency. The plateau in the conductivity spectrum occurs due to the appearance of long-range dc conductivity. At higher frequency, the conductivity becomes more or less with $f_{\mathrm{BaTO}}$ dependent. This frequency at which the change in slope takes place is known as the "hopping or critical frequency $\omega_{\mathrm{H}}$ " and it can be observed that $\omega_{\mathrm{H}}$ increases with increase in $f_{\mathrm{BaTiO}}$ as it can be observed that the length of dc plateau increases with increase in $f_{\mathrm{BaT} \text { TO3 }}$ from 0.3 to 0.6 [34-36].

However, the value of ' $k$ 'lies well within the Johnscher's universal regime $[0,1]$ suggesting the validity of Johnscher's power law in all the PCC universally.

\section{Conclusions}

In conclusion, the microstructural and ac electrical characterisation of a PCC with two component composites, e.g. $\mathrm{PVDF} / \mathrm{n}-\mathrm{BaTiO}_{3}$ prepared through the effective cold pressing method as a function of frequency and temperature and $f_{\mathrm{BaTiO}}$, were investigated. It is found that the enhancement of $\varepsilon_{\text {eff }}$ of PCC based on PVDF matrix is largely enhanced by the cold pressing process conditions, due to the retention of the spherulites during sample preparation. It is also found that this cold pressing method is more suitable to the PCC based on PVDF matrix (since very low value of $\tan \delta \sim 0.9$ is observed) in comparison with PMC based on PVDF matrix in which large value of $\tan \delta \sim 10-100$ is observed. The spherulites present in PVDF matrix are always helpful in maintaining 
the $\varepsilon_{\mathrm{r}}$ and in increasing the $\varepsilon_{\mathrm{eff}}$ of PCC/PMC. The enhancement of dielectric results has been explained with the help of the both interfacial polarisation at the interfaces of the spherulites and the well-established Yamada model to account for the sum effect observed in these PCC. The development of a theoretical work remained as an open challenge to account for both the contribution of interfacial polarisation and the conventional sum effect presents in this type of PCC. A long-range dc conductivity appears at $f_{\mathrm{BaTiO} 3} \geq 0.55$, and the Johnscher's universal fractional power law is well satisfied for all the PCC. The ac hopping conduction, which is the mechanism in these disordered materials have been confirmed in all PCC. We found that the dynamics of charge carriers are $f_{\mathrm{BaTiO} 3}$ and temperature dependent. Again in order to have the applications of PD, the focus of research should be concentrated on still achieving the lower and lower $\tan \delta$, which will increase the dielectric field strength as well as the high energy density, suitable for electrical energy storage applications.

In this work, the use of commercial $n-\mathrm{BaTiO}_{3}$ was decided as a component of the PCC, instead of fresh synthesised $n-\mathrm{BaTiO}_{3}$, because the study was mainly focused on the method of preparation, i.e. the cold pressing method in place of the traditional hot moulding process, and the research was not based on fillers, and it was also found that cold pressing is an effective method in the development of PD based on PVDF. However, in future works, the use of sensitised barium titanate as well as functionalised nano-ceramics will be introduced into the polymer matrix, and the effect has to be studied. Again another important point, we would like to mention that since in the cold pressing method, no unnecessary outside thermal disorder is introduced into the PCC, which leads to the development of a better stabilized sample; hence, it acts as an effective method of preparation of the PD having its own advantages.

Acknowledgements The Authors would like to thank Prof. V. Verma and Dr. R. K. Pandey for providing the dielectric measurement facility and for fitting the Yamada model with Mathematica, respectively.

Funding This study was funded by UGC-BSR project [Grant No: F.30-12/2014(BSR)] and DST (SERB) project [Grant No: SR/FTP/ PS-177/2011].

\section{Compliance with ethical standards}

Conflicts of interest The authors declare that they have no conflicts of interest.

\section{References}

1. Jawaid M, Khan MM (2018) Polymer-based nanocomposites for energy and environmental applications. Woodhead Publishing, Amsterdam

2. Lin Z, Yang Y, Zhang A (2017) Polymer-engineered nanostructures for advanced energy applications. Springer, Switzerland

3. Thakur VK, Kessler MR (2014) Polymer nanocomposites: new advanced dielectric materials for energy storage applications, in advanced energy materials. Wiley, Hoboken

4. Frohlich H (1958) Theory of dielectrics, Chap. 1. Oxford University Press, London

5. Panda M (2018) AIP Conf Proc 110:0429051

6. Panda M, Soni S (2018) Mater Today Proc 110:0429051

7. Li Q, Chen L, Gadinski MR, Zhang S, Zhang G, Li U, lagodkine E, Haque A, Chen LQ, Jackson A, Wang Q (2015) Nature 523:576

8. Li Q, Yao FZ, Liu Y, Zhang G, Wang H, Wang Q (2018) Annu Rev Mater Res 48:3.1

9. Zhang QM, Li H, Poh M, Xia F, Cheng Z-Y, Xu H, Huang C (2002) Nature 419:284

10. Chen Q, Shen Y, Zhang S, Zhang QM (2015) Annu Rev Mater Res 45:433

11. Prateek VK, Thakur RK (2016) Gupta. Chem Rev 116:4260

12. Dang ZM, Yuan JK, Zha JW, Zhou T, Hu GH (2012) Prog Mater Sci 57:660

13. Panda M (2017) Appl Phys Lett 110:0429051

14. Panda M, Srinivas V, Thakur AK (2008) Appl Phys Lett 93:2429081

15. Panda M, Srinivas V, Thakur AK (2008) Appl Phys Lett 92:1329051

16. Panda M, Srinivas V, Thakur AK (2011) Appl Phys Lett 99:0429051

17. Panda M, Srinivas V, Thakur AK (2014) Curr Appl Phys 14:1596

18. Panda M, Srinivas V, Thakur AK (2014) Mod Phys Lett B 28:1450055

19. Panda M (2018) J Adv Dielect 08:1850028

20. Panda $M$ (2010) Scaling and relaxation behaviour of polymermetal composites across the percolation threshold, Ph.D. Thesis, IIT, Kharagpur

21. Panda M, Srinivas V, Thakur AK (2014) J Adv Dielectr 04:1450027

22. Panda M, Srinivas V, Thakur AK (2015) Res Phys 05:136

23. Zhang L, Bass P, Cheng ZY (2014) Appl Phys Lett 105:042905

24. Yang M, Zhao H, He D, Bai J (2016) Appl Phys Lett 109:072906

25. He F, Lau S, Chan HL, Fan J (2008) Adv Mater (Weinheim Ger) 20:A1

26. Dang ZM, Xu HP, Wang HY (2007) Appl Phys Lett 90:012901

27. Bai Y, Cheng ZY, Bharati V, Xu HS, Zhang QM (2000) Appl Phys Lett 76:3804

28. Yamada T, Ueda T, Kitayama T (1982) J Appl Phys 53:4328

29. Zha JW, Dang ZM, Yang T, Zhou T, Song HT, Li ST, Trans IEEE (2012) Dielectr Electr Insul 19:1312

30. Fan BH, Zha JW, Wang D, Zhao J, Dang ZM (2012) Appl Phys Lett 100:012903

31. Chanmal CV, Jog JP (2008) Express Polym Lett 2:294

32. Criest B, Schultz JM (2016) Prog Polym Sci 56:01

33. Stauffer D, Aharony A (1992) Introduction to percolation theory. Taylor and Francis, London

34. Dyre JC, Schroder TB (2000) Rev Mod Phys 72:873

35. Sinclair DC, West AR (1989) J Appl Phys 66:3850

36. Sinclair DC, West AR (1994) J Mater Sci 29:6061

Publisher's Note Springer Nature remains neutral with regard to jurisdictional claims in published maps and institutional affiliations. 